Article

\title{
Using Deep Learning for Image-Based Different Degrees of Ginkgo Leaf Disease Classification
}

\author{
Kaizhou Li®, Jianhui Lin *, Jinrong Liu and Yandong Zhao
}

School of Technology, Beijing Forestry University, Beijing 100083, China; 18810788691@163.com (K.L.); ljr20901@163.com (J.L.); yandongzh@bjfu.edu.cn (Y.Z.)

* Correspondence: swiq_lin@163.com; Tel.: +86-010-6233-6922

Received: 27 December 2019; Accepted: 6 February 2020; Published: 10 February 2020

\begin{abstract}
Diseases from Ginkgo biloba have brought great losses to medicine and the economy. Therefore, if the degree of disease can be automatically identified in Ginkgo biloba leaves, people will take appropriate measures to avoid losses in advance. Deep learning has made great achievements in plant disease identification and classification. For this paper, the convolution neural network model was used to classify the different degrees of ginkgo leaf disease. This study used the VGGNet-16 and Inception V3 models. After preprocessing and training 1322 original images under laboratory conditions and 2408 original images under field conditions, $98.44 \%$ accuracy was achieved under laboratory conditions and $92.19 \%$ under field conditions with the VGG model. The Inception V3 model achieved $92.3 \%$ accuracy under laboratory conditions and $93.2 \%$ under field conditions. Thus, the Inception V3 model structure was more suitable for field conditions. To our knowledge, there is very little research on the classification of different degrees of the same plant disease. The success of this study will have a significant impact on the prediction and early prevention of ginkgo leaf blight.
\end{abstract}

Keywords: Ginkgo leaf disease; classification; deep learning; VGG16; Inception V3

\section{Introduction}

Plant diseases are the main factor endangering the development of agriculture around the world and cause serious losses every year. The treatment of plant diseases has attracted great attention. This research focused on identifying ginkgo leaf disease through leaf blight. Ginkgo biloba has a high medicinal value [1,2]. Its trees can be made into exquisite furniture, and its leaves have ornamental value. Ginkgo leaf blight has brought great losses to the economy, so this research focused on the need to diagnose ginkgo leaf disease in a timely and accurate manner.

Early detection and identification of plant disease is very important, so that people will be able to take appropriate preventive measures as soon as possible [3]. The changes caused by plant diseases are very complex and diverse; however, in traditional agricultural and forestry production, most forest producers judge a disease's species and degree based on their experience in observing plant diseases. This requires forest farmers to have the knowledge and skill to identify disease symptoms. Lack of knowledge will lead to inconsistencies in plant disease identification and incorrect treatment and will ultimately delay the treatment period, which will result in unnecessary economic losses. Even if experts are invited to identify a disease, it will take some time. Therefore, it is necessary to implement an automatic system of plant disease recognition and classification.

In the study of automatic classification of plant diseases, some new techniques have been applied [4].

With the development of computational systems in recent years, more and more computer vision technologies have been applied to the recognition of plant diseases. Rumpf et al. [5] used support vector machines for the early detection and identification of healthy and diseased sugar beet leaves. 
Even using multiple classifications for healthy leaves and diseased leaves showing symptoms of three diseases, the authors achieved an accuracy higher than $86 \%$. Depending on the type and stage of disease, the classification accuracy fell between $65 \%$ and $90 \%$. Similarly, the accuracy of pattern recognition in wheat leaf diseases was classified using a support vector machine and was finished by Tian et al. [6]. The classification module was programmed with three feature sets: color features, shape features, and texture features. The method was flexible, and its recognition rate was high.

Computer vision technology is also widely used in plant species and disease classification and recognition [7]. Some researchers have studied a paper on plant identification using computer vision technology and made a detailed review [8]. One application in the field of computer vision technology is Leafsnap, which identifies tree species using photographs of leaves. Kumar et al. used Leafsnap to identify 184 tree species in the Northeastern United States by extracting features from leaf contours [9]. Their system obtained state-of-the-art performance with the real-world images from the new Leafsnap data set, which is the largest of its kind.

Artificial neural networks (ANNs) are also a common detection method [10-12]. Based on the achievements of modern neuroscience research, an ANN has been proposed [13]. It can make simple judgments by simulating the human brain, so it has been widely used in plant disease detection and recognition. Hati et al. [14] programmed it with 400 leaves from 20 plants and tested 134 leaves, achieving an accuracy of $92 \%$.

Today, deep learning has become the most important detection method. Deep learning is a kind of machine learning that is based on the deep neural network with multiple hidden layers. It improves classification accuracy by building machine learning models with many hidden layers and programming a large quantity of data to extract features. Its basic tool is a convolutional neural network (CNN) [15]. In 2012, Hinton et al. took their CNN to the ImageNet Image Recognition Competition for the first time and won the championship [16], after which it attracted the attention of many researchers. Deep learning has also been introduced to plant species identification. For example, the deep CNN was used to classify white beans, red beans, and soybeans, for which a depth of five layers was determined to be the best [17]. Lee et al. [18] classified 44 species of plants, and their CNN's highest accuracy was $99.6 \%$. A CNN was also applied to plant specimens, and transfer learning was used by Carranza-Rojas et al. [19].

CNNs have been widely used in plant disease identification. For example, P. Ferentinos [20] programmed 25 plants and 58 distinct classes of [plant, disease] combinations and achieved a $99.53 \%$ success rate. Sladojevic et al. [21] pointed out that the model was able to recognize 13 different types of plant diseases from healthy leaves, with the ability to distinguish plant leaves from their surroundings. Mohanty et al. [22] programmed a deep CNN to identify 14 crops and 26 diseases with $99.35 \%$ accuracy. Brahimi et al. [23] programmed nine diseases of tomato leaves and achieved $99.18 \%$ accuracy.

However, most researchers have studied plant diseases in two directions, as follows:

- Classification of diseases in different species of plants [20-22].

- Classification of different diseases in the same plant [23,24].

Few studied the classification of disease degree within the same plant disease, and of those people, only some applied deep learning to the identification of diseased ginkgo leaves. However, it is important to take appropriate preventive measures by predicting the development of plant leaf disease. This research has classified the different degrees of ginkgo leaf disease. A CNN was chosen to classify and recognize the disease degree of ginkgo leaf blight.

The goal of this study was as follows:

- To classify the different degrees of disease in Ginkgo biloba leaves using a deep learning model under laboratory and field conditions that takes into account sunshine, temperature, weather, and other factors.

The rest of the paper is divided into the following parts: Section 2 introduces the methods used, Section 3 discusses the results, and Section 4 presents our conclusions. 


\section{Materials and Methods}

In this research, deep learning was used to recognize the disease classification of Ginkgo biloba leaves. This paper divides the entire process into several sections, which helps to describe it better.

\subsection{Data Set}

Ginkgo leaves were collected under laboratory conditions from Longyuan Huamu Park from 10 to 12 August 2016 and collected under real conditions from 3 September to 31 October 2018. The data set contained leaves classified as healthy, mildly diseased, and severely diseased. The laboratory images were taken by a Canon EOS 550D (19 million pixels) in a black box with 50 watts of natural illumination. The leaves were placed on flat white paper and were photographed from a consistent distance above the black box. The focal length was set to a fixed value, and the camera lens was perpendicular to the blade surface. There were four principles used in selecting the leaves:

- Ginkgo leaves have a more complete shape.

- Ginkgo leaves are flat and easy to photograph.

- Ginkgo leaves' surfaces are clean.

- Ginkgo leaves' periodic disease characteristics are clearly distinguishable.

The shooting process under laboratory conditions is shown in Figure 1.

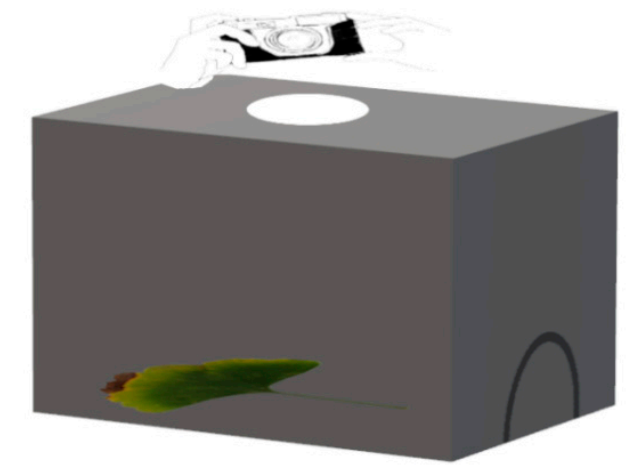

Figure 1. The shooting process under laboratory conditions.

The field photos were taken with a Huawei Honor 7C mobile phone, model LNDAL30 (19 million pixels), under real conditions. When shooting, we placed a piece of white paper on a board to act as the background of the photo. Figure 2 shows samples of a random class containing three representative images (healthy, mild, and severe) under laboratory conditions and three under real conditions. 

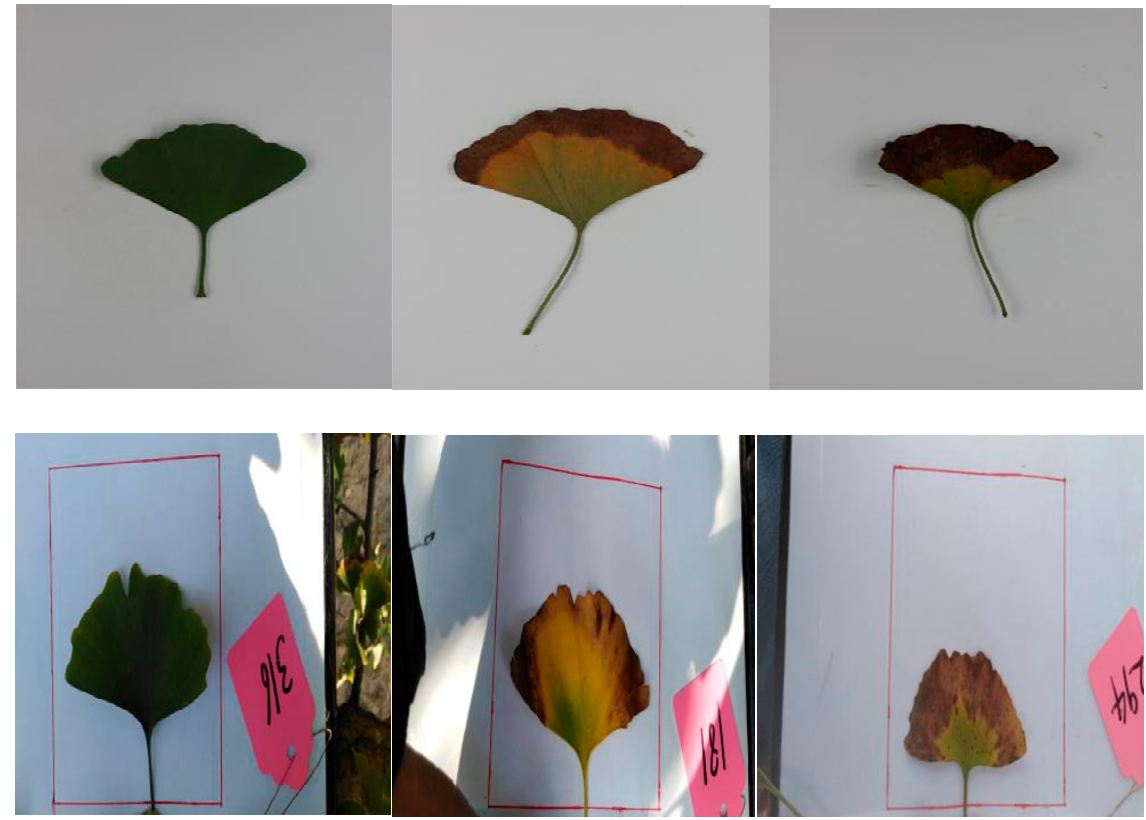

Figure 2. Samples of images under laboratory (top) and field (bottom) conditions.

For this paper, only some of the images were selected from the originals. The number of original images is shown in Table 1.

Table 1. The number of original images.

\begin{tabular}{ccc}
\hline Disease Degree & Laboratory Conditions & Field Conditions \\
\hline Healthy & 326 & 87 \\
Mild & 671 & 1945 \\
Severe & 322 & 376 \\
\hline
\end{tabular}

\subsection{Image Preprocessing and Labeling}

We preprocessed the original images to get better a feature extraction effect, which reduced the time needed for network programming. The digital camera took pictures ranging in size from 1887 to $6770 \mathrm{~KB}$, while the pictures taken with the Huawei mobile phone ranged from 1270 to $2847 \mathrm{~KB}$. The laboratory photos were cut from 1887-2404 KB to 157-540 KB, and their dimensions were reduced from $5184 \times 3456 \mathrm{px}$ to $1800 \times 1200 \mathrm{px}$. Similarly, the field photos were rotated and cut from $1270-2847$ $\mathrm{KB}$ to $298-643 \mathrm{~KB}$, though their dimensions $(4160 \times 2080 \mathrm{px})$ remained unchanged. The process is shown in Table 2.

Table 2. The process of image preprocessing.

\begin{tabular}{ccc}
\hline & Laboratory Conditions & Field Conditions \\
\hline Original picture size (KB) & $1887-6770$ & $1270-2847$ \\
Original picture size (pixels) & $5184 \times 3456$ & $4160 \times 2080$ \\
Experimental picture size (KB) & $157-540$ & $298-643$ \\
Experimental picture size (pixels) & $1800 \times 1200$ & $4160 \times 2080$ \\
\hline
\end{tabular}

\subsection{Data Augmentation}

Although the deep learning neural network is very powerful, if there are not enough data, it will result in overfitting, which cannot achieve the desired results [25]. Many researchers have done a lot of work on this topic; for instance, data augmentation has been used to expand programming images. 
The augmentation methods included image rotation and clipping. After data augmentation, the new data set reached 15,670. Of those, 5569 were healthy, 5964 were mild, and 4137 were severe. The new data set is in Table 3.

Table 3. The number of the new data set.

\begin{tabular}{ccc}
\hline Disease Degree & Laboratory Conditions & Field Conditions \\
\hline Healthy & 5569 & 5569 \\
Mild & 5964 & 5964 \\
Severe & 4137 & 4137 \\
Total & 15,670 & 15,670 \\
\hline
\end{tabular}

\subsection{Convolutional Neural Network Models}

The advantage of an ANN is that it can conduct supervised learning during training. A CNN is a kind of deep feedforward ANN. CNNs have achieved great success in various types of image recognition, including in the field of plant disease diagnosis, and can improve the accuracy of plant disease diagnosis. During recognition and classification, the $\mathrm{CNN}$ can directly input the original image to extract features, thus reducing a lot of preprocessing.

The VGGNet-16 [26] and Inception V3 [27] models were chosen to test the data in this work. Compared with AlexNet, VGGNet increases the network depth and reduces the convolution kernel size, which can reduce the parameters and computation. In addition, the generalization performance of VGGNet is very good. VGGNet-19 has a large number of parameters, so we chose VGGNet-16.

In order to reduce the number of parameters, GoogLeNet model is proposed. It uses the Inception module. Inception V3 introduced factorization, which split a large two-dimensional convolution into two smaller one-dimensional convolutions (e.g., $3 \times 3$ convolutions into $1 \times 3$ convolutions and $3 \times 1$ convolutions). Figure 3 shows the structure of the Inception V3 module.

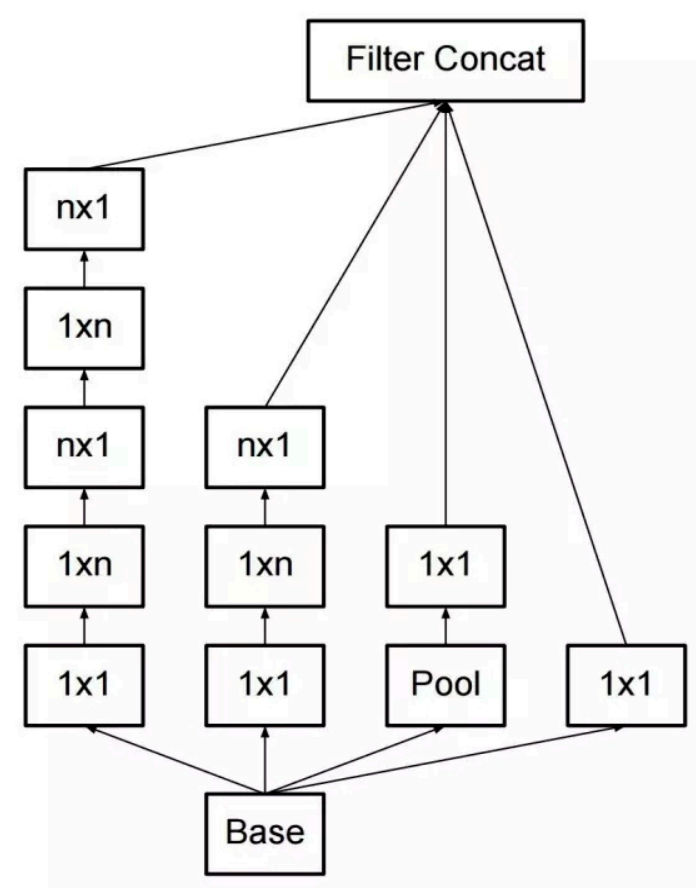

Figure 3. The network structure of Inception V3.

ResNet model has a deeper network. The data we want to train do not need such a deep network. Therefore, we did not choose ResNet model. 
Based on Tensorflow, the VGGNet16 and Inception V3 models were used to program the data of Ginkgo biloba leaves.

\subsection{Training Data Sets}

The data set was divided into two parts: a training set and a test set. Eighty percent of the data were used as a training set to program the network and $20 \%$ as a test set. The images in the VGGNet model were cropped to $224 \times 224 \mathrm{px}$, while the Inception V3 cropped them to $299 \times 299 \mathrm{px}$. Two different CNN models were programmed using the training parameters shown in Table 4.

Table 4. The training parameters of different $\mathrm{CNN}$ models.

\begin{tabular}{ccc}
\hline Parameters & VGG-16 & Inception V3 \\
\hline Batch-size & 64 & 64 \\
Step & 2000 & 4000 \\
Input-width & 224 & 299 \\
Input-height & 224 & 299 \\
Learning & rate & $0.01-0.0001$ \\
\hline
\end{tabular}

\section{Results and Discussion}

This research was programmed with $80 \%$ of the data set and tested with $20 \%$ of the data set. The results are presented in Tables 5 and 6.

Table 5. The results of VGG16 model.

\begin{tabular}{ccccc}
\hline \multirow{2}{*}{ Learning Rate } & \multicolumn{4}{c}{ VGG16 } \\
\cline { 2 - 5 } & \multicolumn{2}{c}{ Laboratory Conditions } & \multicolumn{2}{c}{ Field Conditions } \\
\cline { 2 - 5 } & Accuracy & Loss & Accuracy & Loss \\
\hline 0.01 & $93.75 \%$ & 0.15 & $81.25 \%$ & 0.51 \\
0.005 & $98.44 \%$ & 0.05 & $87.50 \%$ & 0.26 \\
0.001 & $98.44 \%$ & 0.02 & $92.19 \%$ & 0.17 \\
0.0005 & $98.44 \%$ & 0.03 & $89.06 \%$ & 0.24 \\
0.0001 & $98.44 \%$ & 0.05 & $85.94 \%$ & 0.37 \\
\hline
\end{tabular}

Table 6. The results of Inception V3 model.

\begin{tabular}{ccc}
\hline \multirow{2}{*}{ Learning Rate } & \multicolumn{2}{c}{ Inception V3 } \\
\cline { 2 - 3 } & Laboratory Conditions & Field Conditions \\
\cline { 2 - 3 } & Accuracy & Accuracy \\
\hline 0.01 & $92.30 \%$ & $93.20 \%$ \\
0.001 & $88.60 \%$ & $89.00 \%$ \\
0.0001 & $73.40 \%$ & $73.20 \%$ \\
\hline
\end{tabular}

For the VGGNet-16 model in Table 5, the initial learning rates were set to $0.01,0.001$, and 0.0001 . Under laboratory conditions, the accuracy increased gradually and reached $98.44 \%$, then stabilized. Under field conditions, the accuracy increased first and then decreased, which was different from the expected outcome. When the learning rate was 0.001 , the accuracy rate was highest $(92.19 \%)$. To verify whether the learning rate consistently increases first and then decreases, experiments with learning rates of 0.005 and 0.0005 were added. The final results showed that under field conditions, the accuracy increased first and then decreased with the decrease in learning rate. The reason was that when it was lower than 0.001 , the learning rate was too small, resulting in slow convergence rate and parameter update range. Therefore, we should continue to increase the number of training steps in order to make 
the accuracy converge to the best. Figures 4 and 5 show the accuracy curve of the VGG model under laboratory and field conditions.
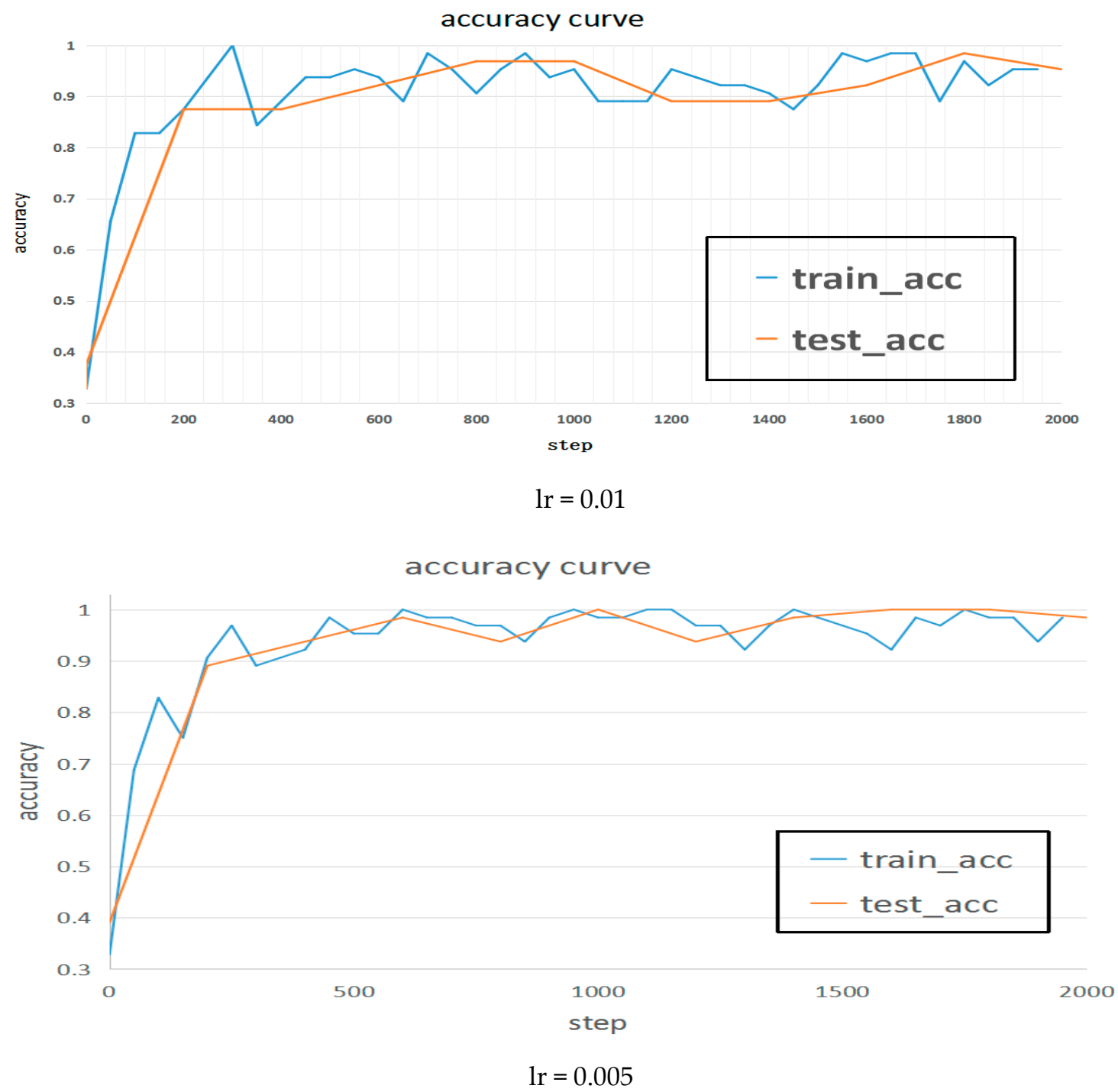

Figure 4. Cont. 

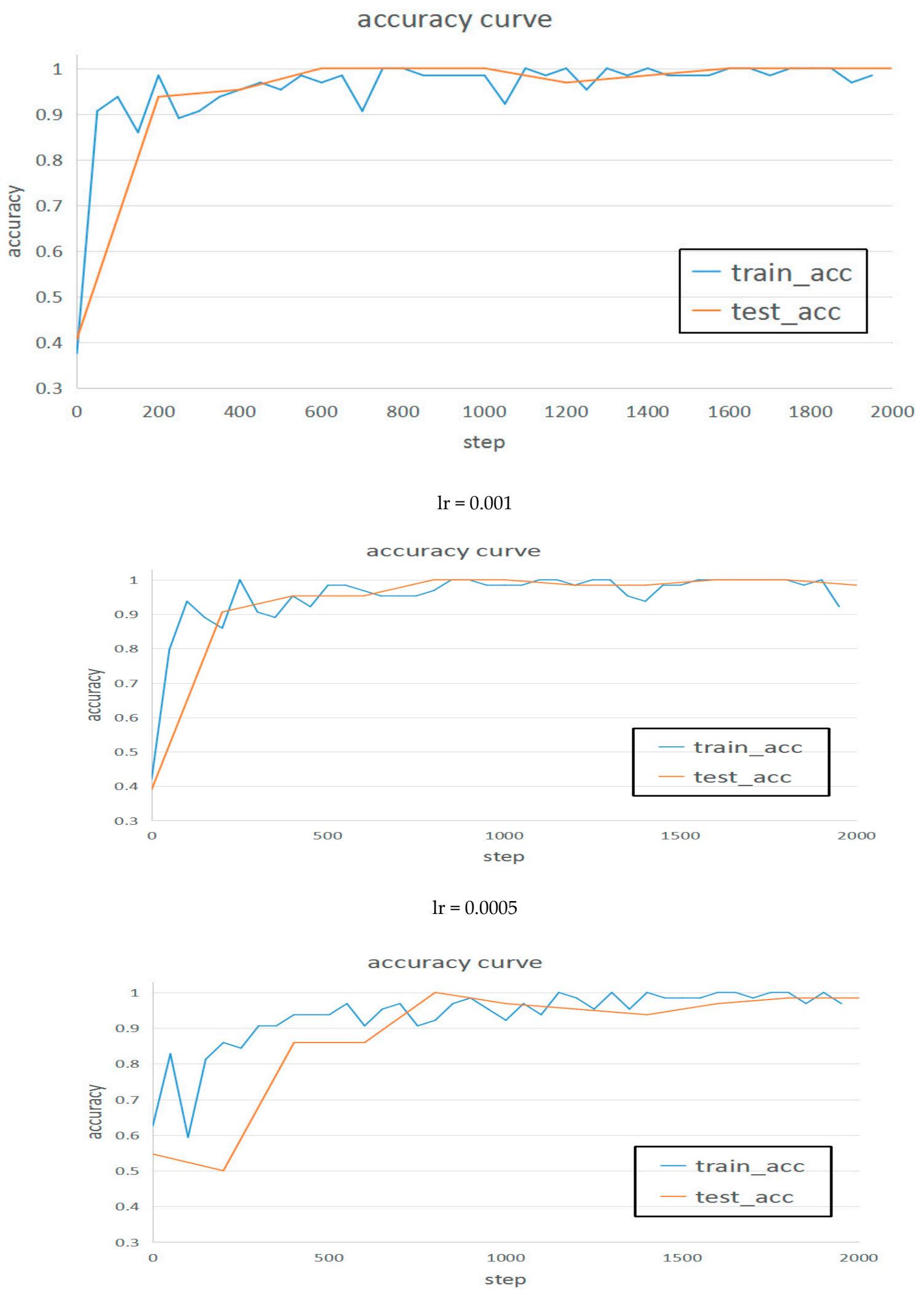

$$
\operatorname{lr}=0.0001
$$

Figure 4. Accuracy curve of VGG model under laboratory conditions. 

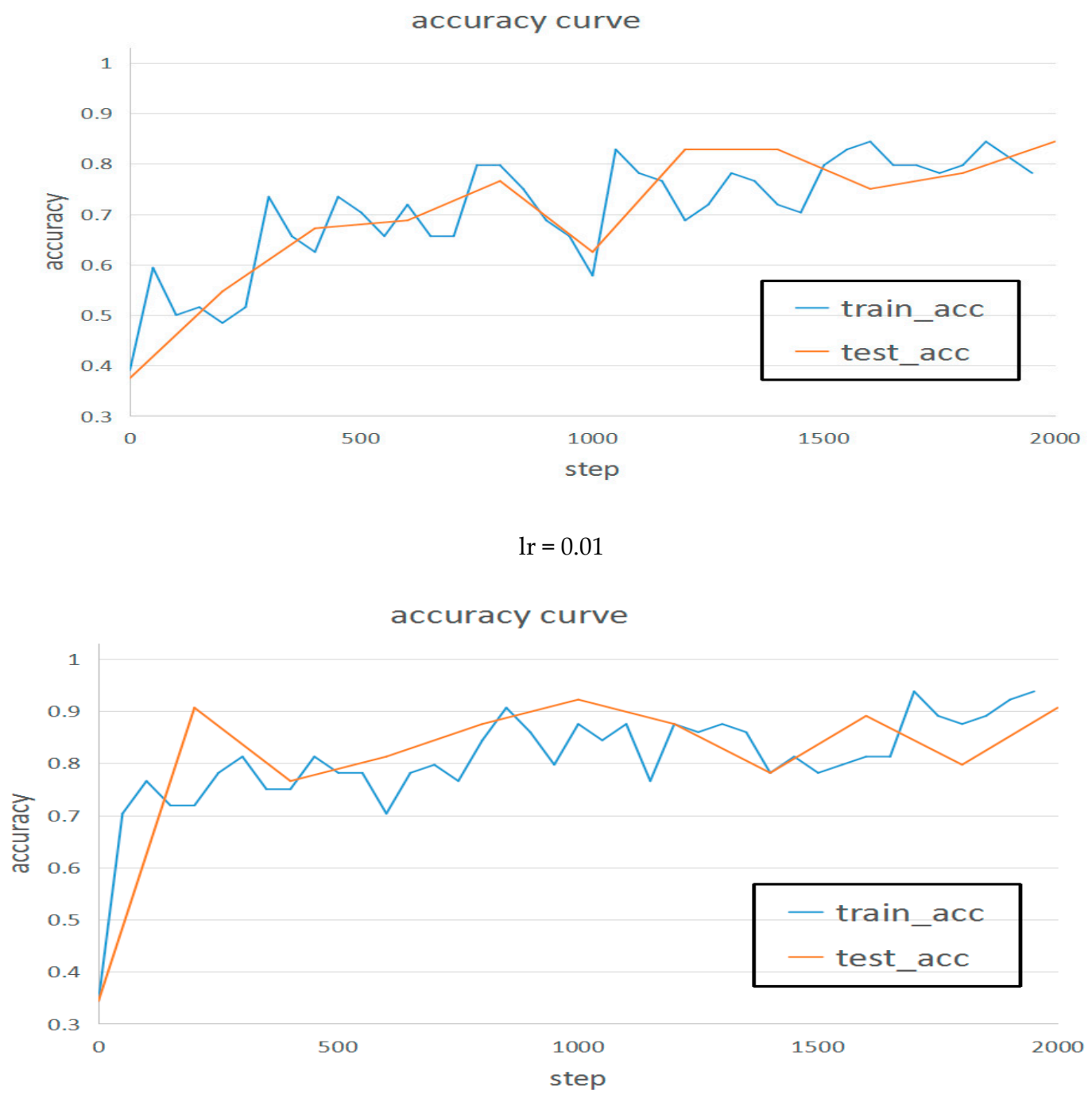

$\operatorname{lr}=0.005$

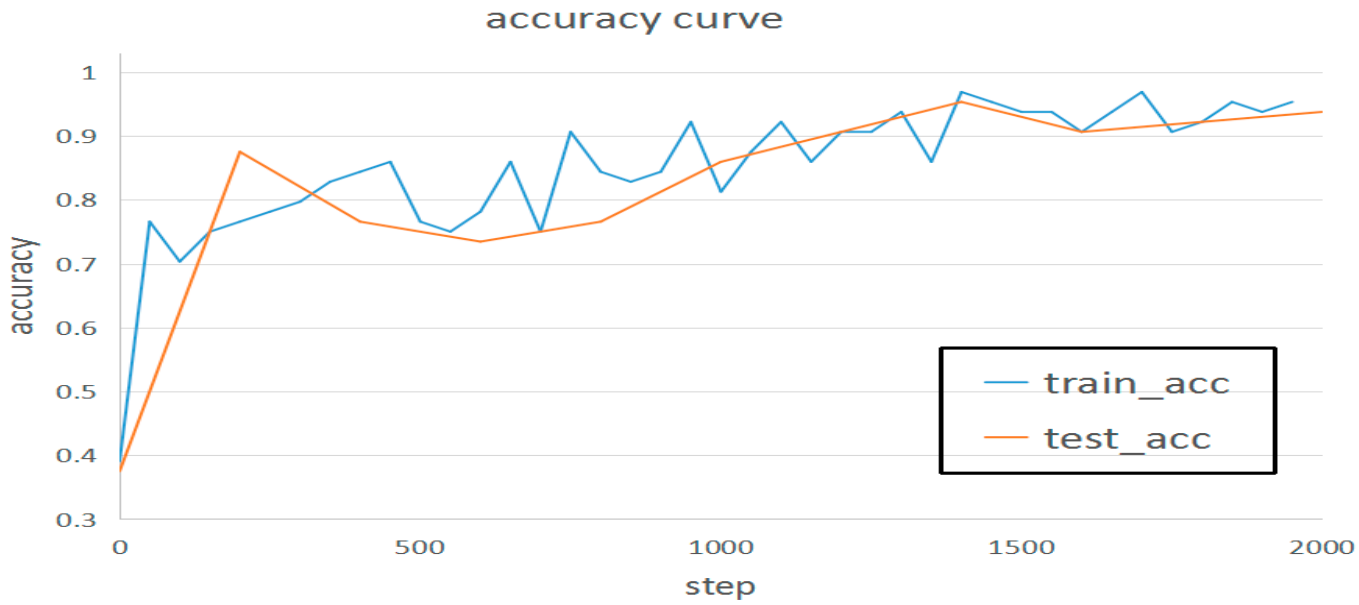

$\operatorname{lr}=0.001$

Figure 5. Cont. 

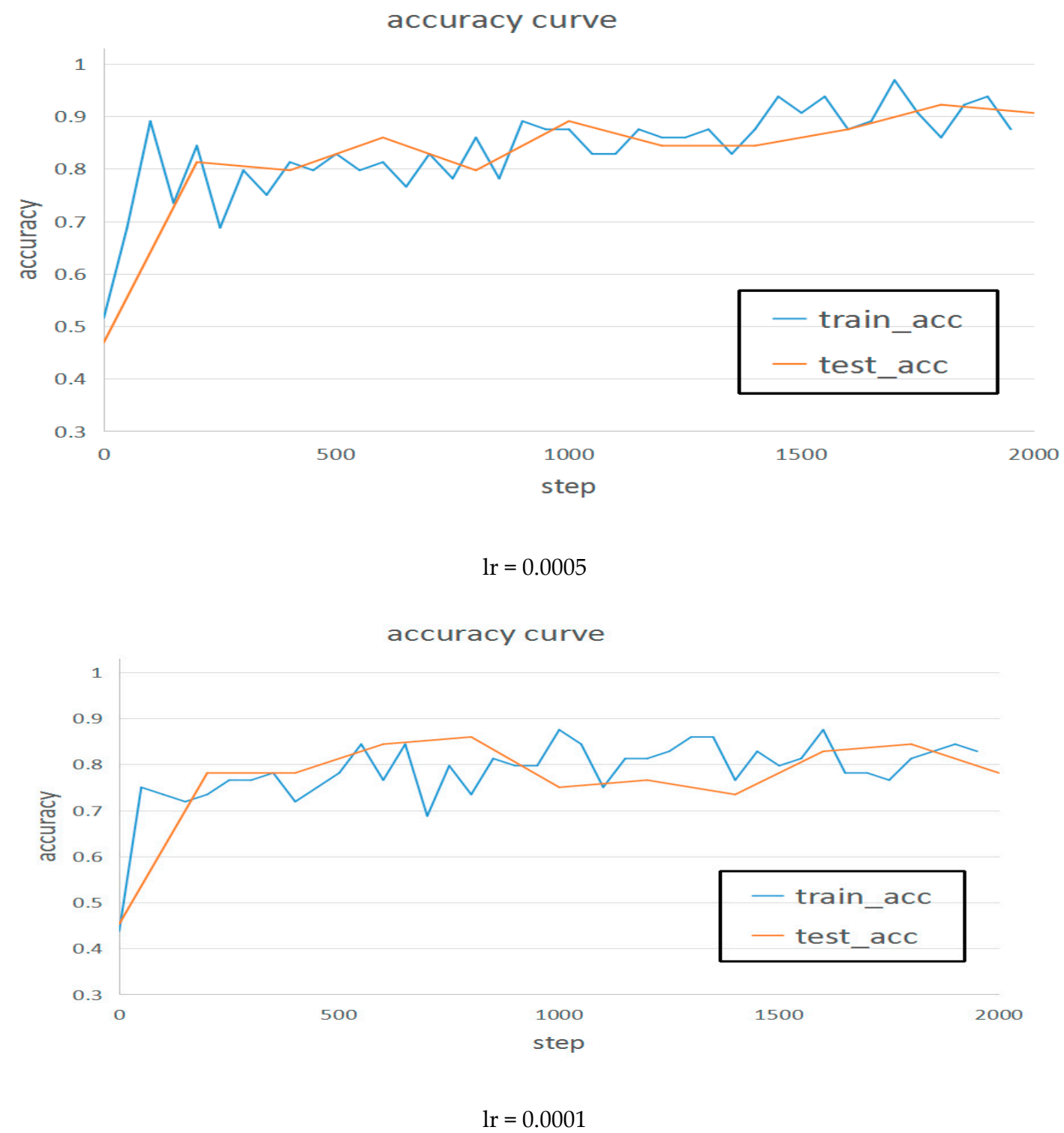

Figure 5. Accuracy curve of VGG model under field conditions.

For the Inception V3 model in Table 6, the initial learning rates were set to $0.01,0.001$, and 0.0001 . Under laboratory and field conditions, the accuracy decreased uniformly. Thus, when the learning rate was 0.01 , the accuracy rate was the highest $(92.3 \%$ and $93.2 \%$, respectively). When it is lower than 0.001 , we should continue to increase the number of training steps in order to make the accuracy converge to the best. Figures 6 and 7 show the accuracy curve of the Inception V3 model under laboratory and field conditions. 

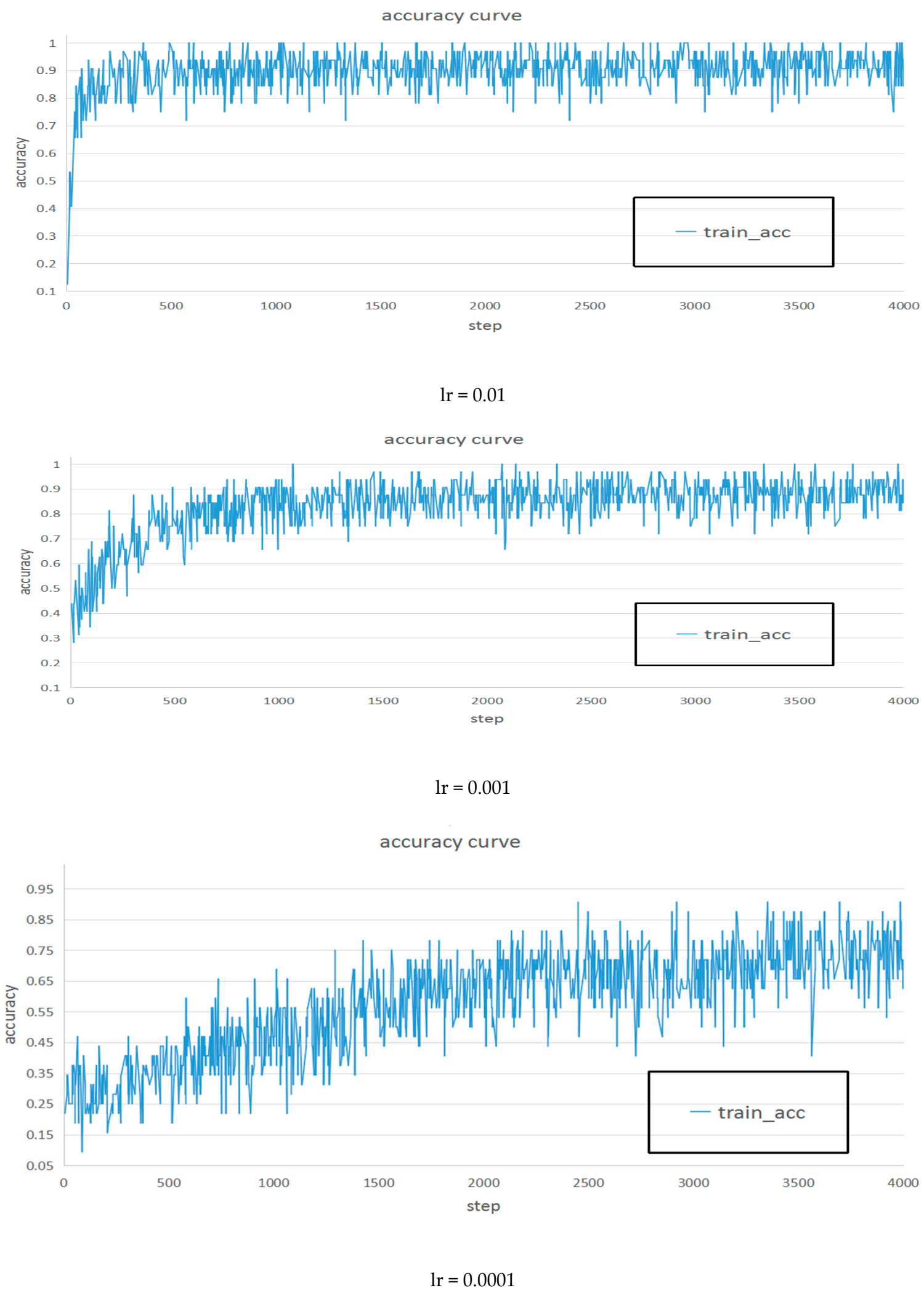

Figure 6. Accuracy curve of Inception V3 model under laboratory conditions. 

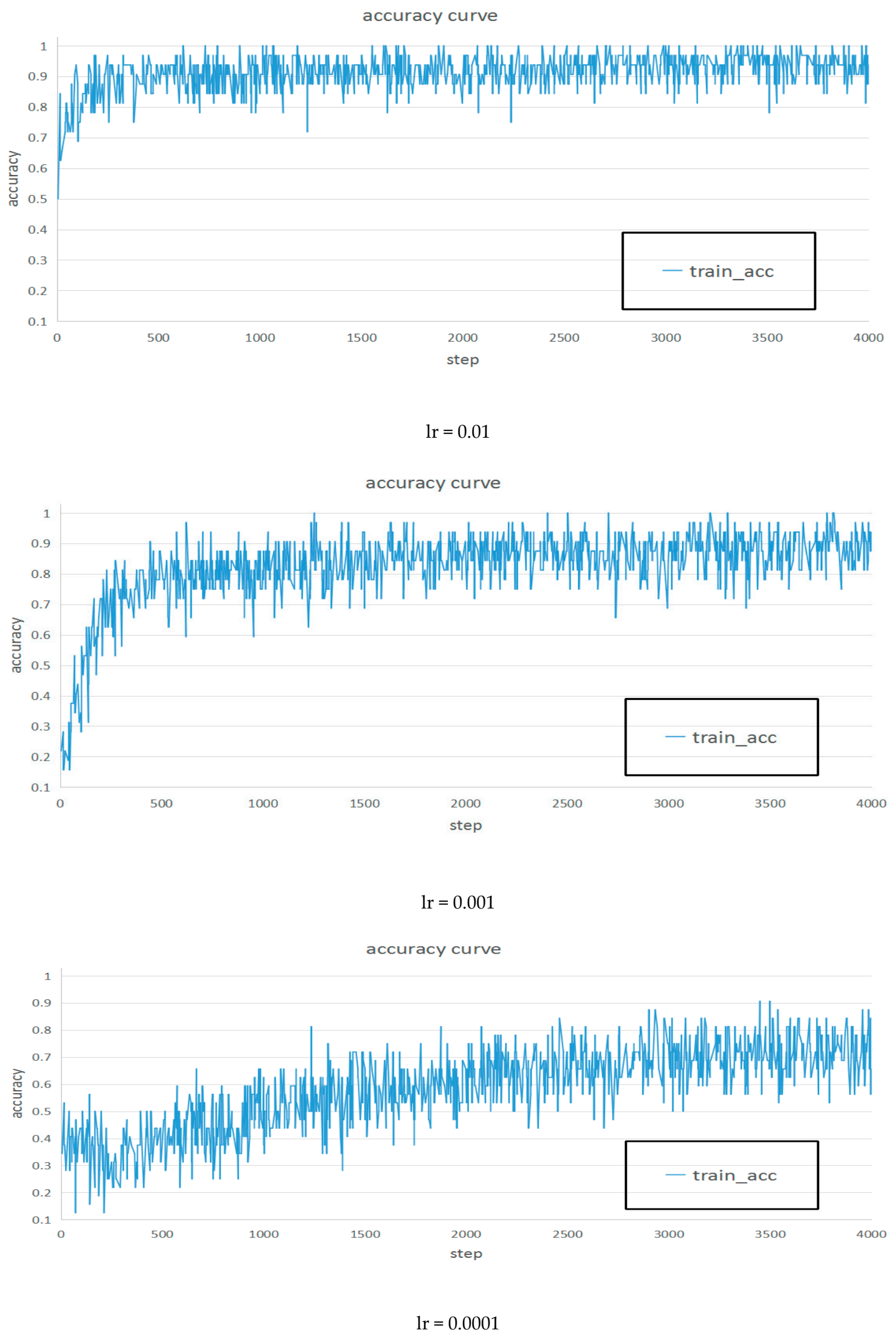

Figure 7. Accuracy curve of Inception V3 model under field conditions. 
Between the two models, the accuracy of the VGG model is higher than that of the Inception V3 model under laboratory conditions, but the accuracy of the Inception V3 model is higher than that of the VGG model under field conditions. For the VGG model, the accuracy under laboratory conditions is higher than it is under field conditions. This demonstrates the fact that classification under field conditions is much more difficult and complex than it is under laboratory conditions. Under field conditions, the increase in network parameters, slow programming, and disappearance of gradient led to a decrease in accuracy. This shows that it is important to capture photos under field conditions to automatically detect and classify plant diseases. For the Inception V3 model, the accuracies of the two conditions were close. The results meant that the Inception V3 model was more stable than the VGG model. With Inception V3, two losses were added at different depths to avoid the disappearance of gradient, and it also used the Inception module. Through these, the depth and width were increased, and the number of parameters was reduced, making the model more stable and adaptable. It can be seen from Figure 7 that the convergence effect of the accuracy curve is better.

Therefore, when we want to classify and recognize diseased leaves under field conditions, we can choose the Inception V3 model. It can be said that the model has achieved good results. There were also many studies that showed the advantages of using deep learning models in plant disease detection and classification [28].

\section{Conclusions}

In this study, a CNN was applied to the field of ginkgo leaf diseases, which have not been studied yet, and classified the different degrees of ginkgo leaf disease. This direction is different from the ones taken by other studies of plant diseases. Of the original images in the data set, 1319 were taken under laboratory conditions and 2408 under field conditions. After pretreatment and data augmentation, the VGG16 and Inception V3 model structures were used to program the data set. The VGG model achieved $98.44 \%$ accuracy under laboratory conditions and $92.19 \%$ under field conditions; however, the results were quite different. On the other hand, the Inception V3 model achieved consistent accuracies under both laboratory and field conditions. This proved that the Inception V3 model structure is more suitable to classify the different degrees of ginkgo leaf disease in field conditions.

The goal of our research has been mostly completed. We believe that with some more work, our findings will have a practical impact on the prevention of disease in ginkgo leaves.

Author Contributions: Conceptualization, K.L. and J.L. (Jianhui Lin); methodology, K.L.; validation, J.L. (Jianhui Lin); formal analysis, K.L. and J.L. (Jianhui Lin); resources, K.L. and J.L. (Jinrong Liu); writing-original draft preparation, K.L.; writing-review and editing, K.L. and J.L. (Jianhui Lin); supervision, Y.Z. All authors have read and agreed to the published version of the manuscript.

Funding: This research was supported by "the Fundamental Research Funds for the Central Universities (NO. 2015ZCQ-GX-03)."

Conflicts of Interest: The authors declare no conflict of interest.

\section{References}

1. Dekosky, S.T.; Williamson, J.D.; Fitzpatrick, A.L.; Kronmal, R.A.; Ives, D.G.; Saxton, J.A. Ginkgo biloba for prevention of dementia: A randomized controlled trial. JAMA. 2008, 300, 2253-2262. [CrossRef] [PubMed]

2. Watanabe, C.M.H.; Wolffram, S.; Ader, P.; Rimbach, G.; Packer, L.; Maguire, J.J. The in vivo neuromodulatory effects of the herbal medicine ginkgo biloba. Proc. Natl. Acad. Sci. USA 2001, 98, 6577-6580. [CrossRef] [PubMed]

3. Sally, A.M.; Beed, F.D.; Harmon, C.L. Plant disease diagnostic capabilities and networks. Annu. Rev. Phytopathol. 2009, 47, 15-38.

4. Sankaran, S.; Mishra, A.; Ehsani, R. A review of advanced techniques for detecting plant diseases. Comput. Electron. Agric. 2010, 72, 1-13. [CrossRef] 
5. Rumpf, T.; Mahlein, A.K.; Steiner, U.; Oerke, E.C.; Dehne, H.W.; Plumer, L. Early detection and classification of plant diseases with Support Vector Machines based on hyperspectral reflectance. Comput. Electron. Agric. 2010, 74, 91-99. [CrossRef]

6. Tian, Y.; Zhao, C.J.; Lu, S.L.; Guo, X.Y. Multiple Classifier Combination For Recognition Of Wheat Leaf Diseases. Intell. Autom. Soft Comput. 2011, 17, 519-529. [CrossRef]

7. Wilf, P.; Zhang, S.; Chikkerur, S.; Little, S.A.; Wing, S.L.; Serre, T. Computer vision cracks the leaf code. Proc. Natl. Acad. Sci. USA 2016, 113, 3305-3310. [CrossRef]

8. Wäldchen, J.; Mäder, P. Plant species identification using computer vision techniques: A systematic literature review. Arch Comput. Method E. 2018, 25, 507-543. [CrossRef]

9. Kumar, N.; Belhumeur, P.N.; Biswas, A.; Jacobs, D.W.; Kress, W.J. Leafsnap: A computer vision system for automatic plant species identification. In European Conference on Computer Vision; Springer: Berlin/Heidelberg, Germany, 2012; pp. 502-516.

10. Wu, Q.F.; Lin, K.H.; Zhou, C.G. Feature extraction and automatic recognition of plant leaf using artificial neural network. Adv. Artif. Intell. 2007, 3, 5-12.

11. Hong, F. Extraction of Leaf Vein Features Based on Artificial Neural Network-Studies on the Living Plant Identification I. Chin. Bull. Bot. 2004, 21, 429-436. (In Chinese)

12. Rastogi, A.; Arora, R.; Sharma, S. Leaf disease detection and grading using computer vision technology and fuzzy logic. In Proceedings of the 2nd International Conference on Signal Processing and Integrated Networks (SPIN), Noida, India, 19-20 February 2015; pp. 500-505.

13. Mehrotra, K.; Mohan, C.K.; Ranka, S. Elements of Artificial Neural Networks. A Bradford Book; The MIT Press: Cambridge, MA, USA; London, UK, 1997.

14. Hati, S.; Sajeevan, G. Plant recognition from leaf image through artificial neural network. IJCA 2013, 62, 15-18. [CrossRef]

15. LeCun, Y.; Bottou, L.; Bengio, Y.; Haffner, P. Gradient-based learning applied to document Recognition. Proc. IEEE 1998, 86, 2278-2324. [CrossRef]

16. Krizhevsky, A.; Sutskever, I.; Hinton, G.E. Imagenet Classification with Deep Convolutional Neural Networks. Adv. Neural Inf. Process. Syst. 2012, 25, 1097-1105. [CrossRef]

17. Grinblat, G.L.; Uzal, L.C.; Larese, M.G.; Granitto, P.M. Deep learning for plant identification using vein morphological patterns. Comput. Electron. Agric. 2016, 127, 418-424. [CrossRef]

18. Lee, S.H.; Chan, C.S.; Wilkin, P.; Remagnino, P. Deep-plant: Plant identification with convolutional neural networks. In Proceedings of the IEEE International Conference on Image Processing (ICIP), Quebec City, QC, Canada, 27-30 September 2015; pp. 452-456.

19. Carranza-Rojas, J.; Goeau, H.; Bonnet, P.; Mata-Montero, E.; Joly, A. Going deeper in the automated identification of Herbarium specimens. BMC Evol. Biol. 2017, 17, 181. [CrossRef]

20. Ferentinos, K.P. Deep learning models for plant disease detection and diagnosis. Comput. Electron. Agric. 2018, 145, 311-318. [CrossRef]

21. Sladojevic, S.; Arsenovic, M.; Anderla, A.; Culibrk, D.; Stefanovic, D. Deep Neural Networks Based Recognition of Plant Diseases by Leaf Image Classification. Comput. Intell. Neurosc. 2016, 2016, 3289801. [CrossRef]

22. Mohanty, S.P.; Hughes, D.P.; Salathé, M. Using Deep Learning for Image-Based Plant Disease Detection. Front Plant Sci. 2016, 17, 1419. [CrossRef]

23. Brahimi, M.; Boukhalfa, K.; Moussaoui, A. Deep learning for tomato diseases: Classification and symptoms visualization. Appl. Artif. Intell. 2017, 31, 299-315. [CrossRef]

24. Lu, Y.; Yi, S.J.; Zeng, N.Y.; Liu, Y.R.; Zhang, Y. Identification of rice diseases using deep convolutional neural networks. Neurocomputing 2017, 267, 378-384. [CrossRef]

25. Hawkins, D.M. The problem of overfitting. J. Chem. Inf. Comput. Sci. 2004, 35, 1-4. [CrossRef] [PubMed]

26. Simonyan, K.; Zisserman, A. Very deep convolutional networks for large-scale image recognition. arXiv 2014, arXiv:1409.1556. 
27. Szegedy, C.; Vanhoucke, V.; Ioffe, S.; Shlens, J.; Wojna, Z. Rethinking the inception architecture for computer vision. In Proceedings of the Computer Vision and Pattern Recognition 2016, Las Vegas, NV, USA, 27 June-28 July 2016; pp. 2818-2826.

28. Schmidhuber, J. Deep Learning in neural networks: An overview. Neural Netw. 2015, 61, 85-117. [CrossRef] [PubMed]

(C) 2020 by the authors. Licensee MDPI, Basel, Switzerland. This article is an open access article distributed under the terms and conditions of the Creative Commons Attribution (CC BY) license (http://creativecommons.org/licenses/by/4.0/). 